\title{
¿Y si quiero regresar a mi país? \\ Niños estadounidenses en escuelas de Cuernavaca
}

\author{
Rodrigo Aguilar Zepeda*
}

Recepción: 13 de septiembre de 2017 Aceptación: 19 de enero de 2018

Resumen El objetivo de este trabajo es analizar el proceso de integración escolar de niños con experiencia migratoria en Estados Unidos que en 2016 residían en la ciudad de Cuernavaca, en el estado de Morelos. El análisis se hace a través de una serie de entrevistas realizadas en cuatro secundarias, en las cuales se analizan la trayectoria migratoria y la trayectoria educativa. Los resultados sugieren que las trayectorias migratorias no son homogéneas y predominan las que son de larga de data, esto no tiene efectos negativos en su proceso de integración escolar. Además se encuentra que la migración por sí misma no afecta la trayectoria escolar de los menores entrevistados, sin embargo el idioma sí afecta el proceso de llegada a la escuela.

PALABRAS CLAVE: menores migrantes, integración escolar, educación, migración de retorno, Cuernavaca.

\section{If I want to return to my country? American children in Cuernavaca schools}

Abstract The objective of this work is to analyze the process of school integration of children with migratory experience in the United States who in 2016 lived in the city of Cuernavaca, in the state of Morelos. The analysis is made through a series of interviews carried out in four middle school, in which the migratory trajectory and the educational trajectory were analyzed. The results suggest that migratory trajectories are not homogeneous and those that are long data predominate, this does not have negative effects on their

Profesor de tiempo completo en el Centro de Investigación y Estudios Avanzados de la Población, Universidad Autónoma del Estado de México, rodrigoagze@gmail.com. Este trabajo de investigación fue posible gracias al apoyo del Programa Binacional de Educación Migrante (PROBEM) del estado de Morelos. 
school integration process. It is also found that migration itself does not affect the educational trajectory of the children interviewed, however the language does affect the process of arriving to school.

KEY WORDS: migrant children, school integration, education, return migration, Cuernavaca.

\section{Introducción}

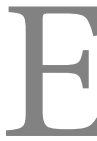

1 contexto actual de la migración de México a Estados Unidos atraviesa por un periodo de incertidumbre y tensión permanente, en especial entre aquellos migrantes mexicanos que residen en Estados Unidos en condición de indocumentados. La entrada de una nueva administración con acciones -eliminación del Programa de Acción Diferida (DACA, por sus siglas en inglés- y discursos antiinmigrantes provoca temor e incertidumbre entre los migrantes. Esto podría provocar que una cantidad considerable de mexicanos - y sus familias- que residen en Estados Unidos decidan regresar a México o sean sujetos de deportación por parte del gobierno estadounidense. Para 2015, se estimó que había 5.6 millones de mexicanos residiendo de manera no autorizada en aquel país, con una disminución de 800 mil con respecto a los 6.4 millones que vivían en las mismas condiciones en 2009 (Passel y Cohn, 2017). Esta etapa coincide con la administración Barack Obama en Estados Unidos, la cual removió a un total de 1.9 millones de mexicanos de 2009 a 2015, es decir, 280 mil por año en promedio. La combinación de factores y tomando en cuenta que el retorno no se realiza, en la mayoría de los casos, en forma individual sino colectiva - familiar-, se está ante el reto de recibir, incorporar e integrar a población que probablemente tiene mucho tiempo viviendo fuera de México o incluso nunca ha vivido aquí, por ejemplo los hijos de los migrantes que nacieron en Estados Unidos. Es esta población en específico la que genera interés en esta investigación. ¿Cuántos son estos menores de edad que han vivido en Estados Unidos - ya sean nacidos en México o en aquel país - y ahora viven aquí? Dada su edad, ¿están asistiendo a la escuela? Si lo hacen, ¿qué dificultades han encontrado en la inserción escolar? ¿Cómo es el proceso de integración de estos menores con experiencia migratoria? Precisamente es esta última pregunta la que esta investigación busca responder.

Por lo tanto, es en este contexto de retorno ${ }^{1}$ forzado y voluntario - por supuesto, más familiar que individual- que esta investigación se propone como objetivo

1 La migración de retorno, entendida como parte del proceso migratorio en la cual una persona regresa a su país de nacimiento después de haber vivido por un periodo de tiempo en un país distinto a aquel donde nació. 
analizar el proceso de integración escolar de niños con experiencia migratoria en Estados Unidos que en 2016 residían en Cuernavaca, Morelos. El análisis se hace por medio de una serie de entrevistas realizadas en cuatro secundarias de Cuernavaca. En ellas se analiza la trayectoria migratoria y la trayectoria educativa como ejes principales que permitan entender los procesos de integración escolar. La elección de esta ciudad se inscribe en una investigación de mayor calado que busca comparar los procesos de integración de menores con experiencia migratoria en las ciudades de Tijuana y Cuernavaca. Para esta investigación se prefirió analizar y profundizar en la población de menores entrevistados en un espacio emergente de migración de retorno.

El presente artículo se divide en tres secciones. En la primera se presenta una discusión sobre la integración en contextos migratorios. La segunda aborda el contexto de retorno al cual llegan los niños con experiencia migratoria. En la tercera se presentan los resultados del análisis de las entrevistas en lo que a la integración escolar se refiere. Además de las secciones referidas, se cuenta con esta introducción y una sección de reflexiones.

\section{Discusión teórica}

El proceso de asimilación a las sociedades receptoras ha sido abordado ampliamente en los contextos de inmigración. Por ejemplo, Portes y Zhou (1993) introducen el concepto de asimilación segmentada para describir los diversos resultados del proceso de adaptación a una nueva sociedad. Se comienza a introducir la idea de que el proceso de adaptación a un nuevo espacio social no es lineal y que puede ser influenciado por el contexto social, político y familiar. En este mismo sentido - que la asimilación no es un proceso lineal-, Rumbaut (2004) hace su propuesta de generaciones decimales, que tiene que ver con la edad en que se realizó la migración. De esta forma, Rumbaut llama generación 1.25 a quienes emigraron en su infancia temprana (de 0 a 5 años), 1.5 a aquellos que emigraron en su infancia media (de 6 a 12 años) y generación 1.75 a los que llegaron en la adolescencia (de 13 a 17 años). Los resultados del proceso de adaptación varían en función de la edad de llegada. Esto se explica porque mientras más pequeño sea al efectuarse la migración más tiempo se está expuesto a la sociedad receptora y, por su puesto, a sus instituciones, como lo es la escuela. Por consiguiente, haber emigrado a mayor edad implica que se estuvo expuesto por más tiempo a las instituciones del punto de partida, lo que puede generar cierto choque entre lo ya adquirido y lo que está por adquirirse. Las propuestas que desde los espacios receptores se han construido y que hacen referencia al proceso de adaptación a la sociedad se han realizado con la población en general; en 
este caso y para esta investigación, se pretende indagar en este proceso en la población menor de edad.

Con lo anterior como respaldo, desde México se han hecho esfuerzos por caracterizar a la población de menores migrantes principalmente en su relación con la educación y desde distintas perspectivas disciplinarias. Desde la sociología, Zúñiga y Hamman (2008) abordan el desafío de las escuelas ante la presencia de alumnos transnacionales - les llaman así a aquellos con experiencia migratoria-; muestran cómo las escuelas y los alumnos están ante un reto dada la diferencia entre sistemas educativos, el mexicano y el estadounidense. Dichos retos se inscriben en los contenidos y desde las visiones que se enseñan hasta las condiciones materiales y de infraestructura de las escuelas en ambos países. Mencionan que son los alumnos con trayectoria transnacional quienes enfrentan esos retos. Otra aportación desde esta perspectiva sociológica es la que hacen Sánchez y Zúñiga (2010); a partir del análisis de trayectorias migratorias de alumnos transnacionales, detectan que estas son diferenciadas y fragmentadas, es decir, no hay una trayectoria única, y la fragmentación se basa en la cantidad de viajes que han realizado entre México y Estados Unidos.

Desde una perspectiva sociodemográfica, Giorguli y Gutiérrez (2011) destacan la participación y exposición de niños y adolescentes en la migración entre México y Estados Unidos, esto las lleva a estimar esta población expuesta a la migración. La importancia de esto radica en que se logra diferenciar a los menores de edad que viven la migración en forma directa de quienes la experimentan indirectamente —alguno de los miembros de su hogar participan en ella-. Una razón más es que hace visible a una población que estaba fuera del análisis de la migración de México a Estados Unidos. Por su parte, Vargas y Camacho (2015) destacan que la migración de retorno tiene un vínculo negativo con la trayectoria educativa de los niños que participan activamente en este tipo de movilidad, en especial entre aquellos nacidos en México, lo que provoca mayor inasistencia y rezago escolar. Resultados similares encuentra Aguilar (2014) al mencionar que hay una mayor desventaja en el rezago escolar entre los niños migrantes de retorno y aquellos que no migraron, la desventaja en el rezago se agrava entre los niños nacidos en México y que habían tenido experiencia de vivir en Estados Unidos. Por último, en un ámbito educativo legal, Jacobo (2017) discute los alcances y las limitaciones de los cambios en las normas en México que facilitan el acceso escolar de los estudiantes mexicoamericanos y profundiza en una caracterización demográfica de los estudiantes mexicoamericanos y en los retos que enfrentan en su inclusión educativa.

Este breve recorrido se presentó con la finalidad de mostrar los elementos teóricos y las evidencias empíricas que se han escrito sobre el tema de interés 
particular en esta investigación, la migración de menores de edad y su proceso de integración escolar.

\section{El contexto de retorno y las definiciones}

La ciudad de Cuernavaca, capital del estado de Morelos, está ubicada en el norte de la entidad y tiene una estrecha cercanía con la Ciudad de México. Según datos de la Encuesta Intercensal 2015 (INEGI), en ella habitan 366,321 personas, que representan el $19.2 \%$ de la población estatal, y este municipio es el más poblado del estado. Morelos no se ha caracterizado por tener un alto volumen de migrantes a Estados Unidos en el contexto nacional, en 2015 registraba un total de 23,845 personas con antecedentes de haber vivido o nacido en aquel país, y solo en Cuernavaca se contabilizaron 3,199 personas, que equivalen al $13.4 \%$ de la población total del estado. Cabe señalar que aun cuando este porcentaje es relativamente bajo, Cuernavaca es el municipio, a nivel estatal, con mayor concentración de personas con antecedentes migratorios en Estados Unidos. Estas características hacen del municipio un espacio propicio para explorar el proceso de integración escolar de niños con experiencia migratoria procedentes de ese país.

ESQUEMA 1. Población total y población con experiencia migratoria en Estados Unidos,

Cuernavaca, 2015

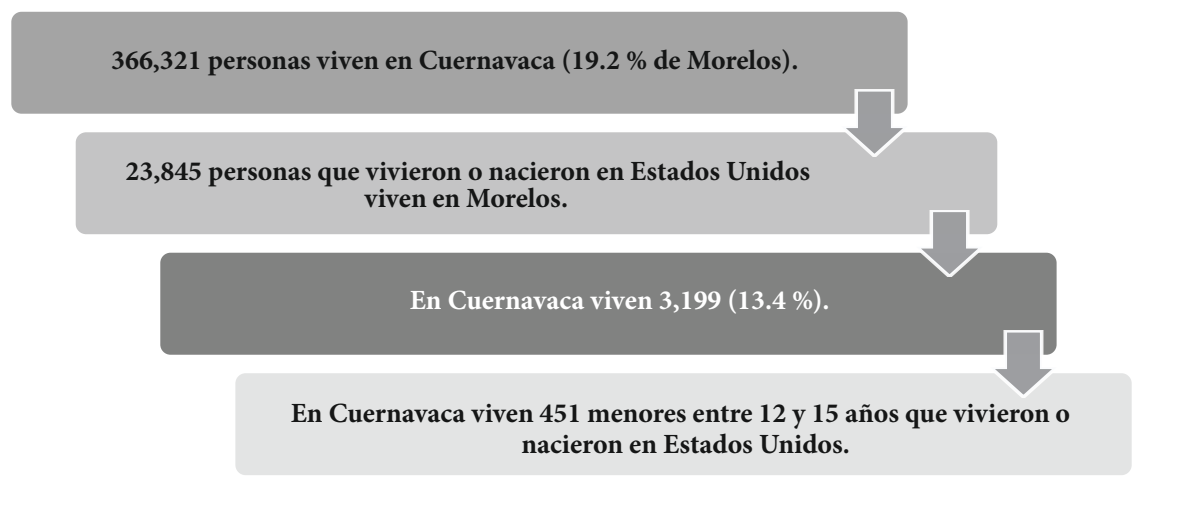

Fuente: Elaboración propia con base en la muestra de la Encuesta Intercensal 2015, INEGI.

En cuanto a los niños a los cuales se entrevistó para esta investigación, ellos cursaban alguno de los años que conforman el nivel de secundaria (nivel básico). En total fueron 12 mujeres y 14 hombres y sus edades oscilaban entre los 12 y 15 años, los detalles de sus características se presentan más adelante. Por otro lado, con la 
finalidad de contextualizar a los menores entrevistados, se muestra un panorama general de la población en ese rango de edades y que además cuenta con experiencia migratoria en Estados Unidos, esto con datos provenientes de la Encuesta Intercensal. De esta forma, en 2015, la población total de entre 12 y 15 años que vivía en Cuernavaca y tenía antecedentes de haber vivido o nacido en Estados Unidos era de 451 menores, esto equivale al $2.1 \%$ del total de población que vive en Cuernavaca en ese mismo rango de edad. Si bien es una población que en volumen y en términos relativos es pequeña, no es una población de la cual se conozcan a fondo sus necesidades particulares; de ahí la importancia de ahondar en al menos dos características, la asistencia escolar y la condición de actividad. La primera característica es una variable que permite observar el acceso a la escuela, condición necesaria para que haya integración escolar. La segunda permite mostrar la relación de esta población con el trabajo y si este es competencia para la continuidad escolar.

Antes de analizar los resultados de la parte contextual de la población estudiada conviene definir quiénes son los niños con experiencia migratoria en Estados Unidos. En esta forma, la definición entrelaza el lugar de nacimiento y la residencia en Estados Unidos. A partir de estas dos variables se construyen tres trayectorias migratorias en las cuales el tiempo de residencia en México aparece como un elemento que las define (esquema 2). La primera corresponde a los niños retornados, ellos son nacidos en México y llevan cinco años o menos viviendo en el país. La segunda corresponde a los niños inmigrantes recientes, ellos son nacidos en Estados Unidos y llevan cinco años o menos viviendo en México. La tercera corresponde a los niños inmigrantes, ellos son nacidos en Estados Unidos y tienen cinco años o más viviendo en el país. A partir de estas tres trayectorias se analiza la población con experiencia migratoria en Estados Unidos que vive en Cuernavaca.

La importancia de distinguir estas tres trayectorias migratorias va de la mano con la evidencia empírica encontrada en el trabajo de campo realizado en las escuelas secundarias de Cuernavaca y que tiene que ver con el tiempo de llegada a la ciudad. Si bien a partir de la Encuesta Intercensal no es posible establecer con exactitud el tiempo de llegada a México, sí permite aproximarse al tiempo que llevan viviendo en el país. En investigación que relaciona trayectorias migratorias y escolares con la integración escolar, el tiempo pasa a ser un factor que permite explicar el proceso de integrarse a un entorno distinto al previamente conocido. Entre la población menor de edad es importante considerar dónde se llevó a cabo la socialización temprana (López Castro, 1999, 2007), por ello el tiempo de estancia en México es una variable que permite aproximarse a qué tan expuestos han estado al sistema escolar mexicano.

Una de las hipótesis que está presente es que un mayor tiempo de exposición a la sociedad en la que se vive la primera infancia hará más complejo el proceso de 
ESQUEMA 2. Definición de la población con experiencia migratoria en Estados Unidos

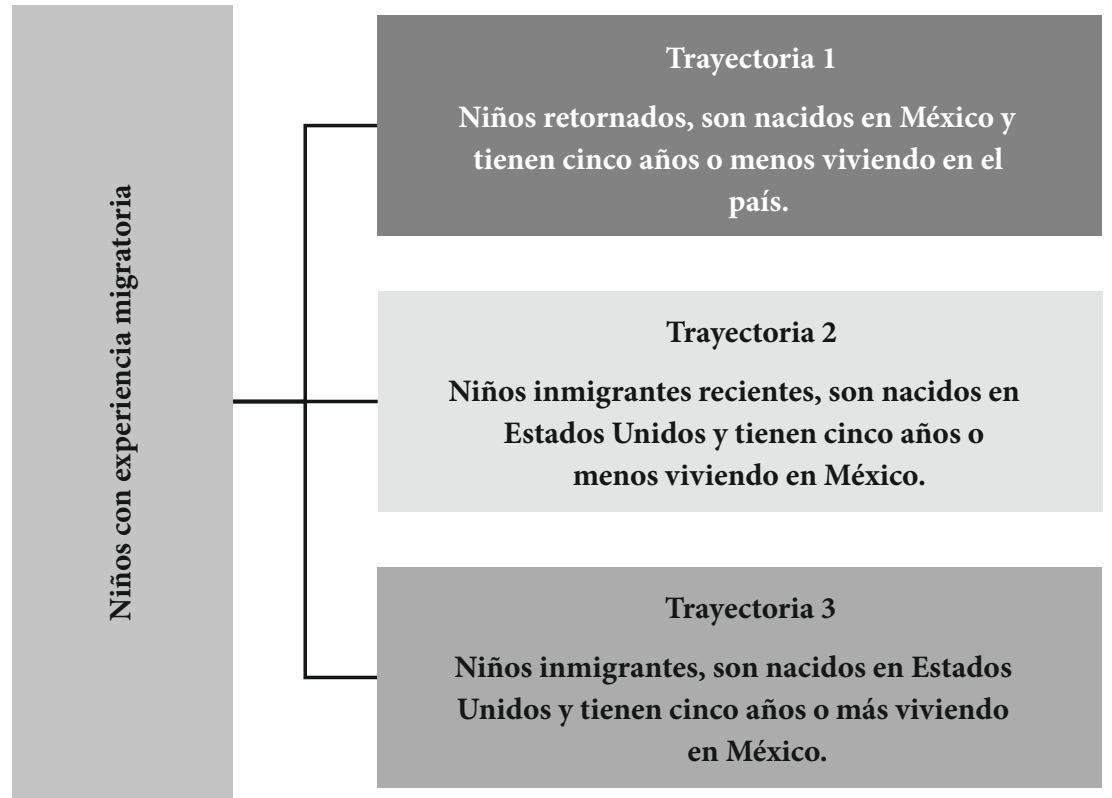

Fuente: Elaboración propia.

integración a la nueva sociedad. En este mismo sentido es que Rumbaut hizo su propuesta de generaciones decimales $(1.12,1.5$ y 1.75), en la que se distingue el proceso de adaptación en función de la edad de llegada. Si bien toda esta población entra en la categoría de menores de edad, la comprobación de esta propuesta en forma empírica hecha por Oropesa y Landale (1997) encuentra diferencias significativas en cuanto a los resultados de adquisición del lenguaje; es decir, ahí la edad en la que se realizó la migración marca la diferencia entre ser bilingüe o monolingüe. Sin duda el lenguaje es un vehículo que permite llevar a cabo la integración en forma tersa o más complicada, en especial cuando no se tiene como habilidad adquirida al momento de la llegada a la sociedad receptora. Cabe señalar que esta propuesta se hace en un contexto de inmigración, pero resulta esclarecedor que pueda servir de guía en contextos de migración de retorno. Hay que recordar que en estricto sentido la mayoría de los menores con experiencia migratoria no son retornados; sin embargo, forman parte de un proceso de retorno colectivo, es decir, vuelven al país de origen de sus padres acompañando su retorno; es por ello que se considera que no es una movilidad migratoria individual, sino colectiva. En el caso particular de los menores de edad en Cuernavaca no se analiza el proceso de integración a la sociedad 
en su conjunto, solo a una parte de ella, y el mecanismo elegido es la escuela, que es un espacio de socialización para los niños migrantes. En esta forma el proceso de integración escolar es entendido como el camino que recorren los menores en su adaptación social, cultural y de aprendizaje en la escuela.

Ahora bien, la importancia de definir a la población migrante a partir del tiempo de estancia en México permite detectar necesidades puntuales de acuerdo con cada tipo de trayectoria, como lo detectan Oropesa y Landale (1997) al poner a prueba la propuesta de Rumbaut. Antes de continuar con los resultados de las trayectorias y el proceso de integración escolar conviene presentar, en forma breve, algunas características generales de los niños con experiencia migratoria. En el rango de edad que corresponde al nivel de secundaria no se detectó, desde la Encuesta Intercensal, a ningún niño con trayectoria de retorno y se identificó a pocos con trayectoria de inmigración reciente; de hecho la mayoría de los niños identificados en la ciudad pertenecen a la trayectoria de niños inmigrantes, es decir, de aquellos que llevan más de cinco años residiendo en México -Cuernavaca-. Dado el pequeño número de casos en la muestra de la Encuesta Intercensal, en las características del grupo de niños de entre 12 y 15 años de edad que se presentan a continuación no se distingue la trayectoria migratoria a la que pertenecen, el único factor común en su caso es haber nacido en Estados Unidos. Cabe recalcar que estos resultados no pretenden ser concluyentes, sino solamente un marco de referencia para la interpretación de las entrevistas realizadas a 26 menores con experiencia migratoria en cuatro secundarias de Cuernavaca, Morelos.

Una de las variables de las cuales es importante dejar registro es la asistencia escolar, en el caso de la población con experiencia migratoria quienes asisten son 93.6 \%; entre la población del mismo rango de edad que vive en Cuernavaca y no son migrantes la asistencia es de $94.6 \%$ (cuadro 1). A nivel nacional la tasa de asistencia a la secundaria es de $94.6 \%$ (INEE, 2016). Es decir, si bien la asistencia escolar de los niños con experiencia migratoria es más baja que sus contrapartes en Cuernavaca e incluso que el promedio nacional, la diferencia observada es mínima. Esto hace suponer que la migración no afecta en forma contundente la asistencia escolar, muy probablemente lo anterior está relacionado con la edad de llegada a México. Para entender a profundidad estos resultados es conveniente el análisis de las trayectorias migratorias que surgieron en las entrevistas con niños de secundaria.

Un dato más que conviene explorar es la condición de actividad de los niños con experiencia migratoria. Los resultados indican que el 7 \% de los niños con experiencia migratoria trabajaron, entre los niños de la misma edad en Cuernavaca que no son migrantes la proporción fue de 5.5 \% (cuadro 1). Es decir, hay una ligera tendencia a que los niños con experiencia migratoria trabajen. 
CUADRO 1. Porcentaje de población de 12 a 15 años por asistencia escolar y condición de actividad según condición migratoria, Cuernavaca, 2015

\begin{tabular}{|c|c|c|}
\hline & Cuernavaca & $\begin{array}{c}\text { Experiencia } \\
\text { migratoria }\end{array}$ \\
\hline & \multicolumn{2}{|c|}{ Asistencia escolar } \\
\hline \multirow[t]{2}{*}{ Sí } & 94.6 & 93.6 \\
\hline & \multicolumn{2}{|c|}{ Trabajó } \\
\hline Sí & 5.5 & 7.1 \\
\hline
\end{tabular}

Fuente: Elaboración propia con base en la muestra de la Encuesta Intercensal 2015, INEGI.

Si bien estas dos variables analizadas perfilan cierta desventaja de los menores con experiencia migratoria en Estados Unidos respecto de aquellos que no la tienen - menor asistencia escolar y mayor trabajo-, no termina por ser un resultado concluyente porque son mínimas las diferencias encontradas. Es decir, no hay una desventaja en el acceso escolar y el trabajo por el hecho de tener o no experiencia migratoria. De esta manera, en el siguiente apartado se presenta la metodología cualitativita, se analiza y profundiza en los resultados de las entrevistas con niños de cuatros escuelas secundarias de Cuernavaca.

\section{El proceso de integración a través de trayectorias migratorias y educativas}

La aproximación cualitativa, a través del análisis de trayectorias - migratorias y escolares- permite profundizar en el proceso de integración de niños migrantes en las escuelas de Cuernavaca. En total se visitaron cuatro secundarias ubicadas en distintos puntos de la ciudad; ${ }^{3}$ esto permitió tener acceso a niños con experiencia migratoria en distintos contextos de llegada (aun cuando están en la misma ciudad), dado que dos de ellas se ubican en el norte de Cuernavaca y las otras dos en el sur. Si bien las cuatro se encuentran en un entorno urbano, dos de ellas responden a un entorno rural inserto en la ciudad. Todas pertenecen al turno matutino. En las cuatro secundarias se logró entrevistar a un total de 26 alumnos, 12 mujeres y 14 hombres. Todos ellos entre los 12 y 15 años de edad, cursando el primero (11), el segundo (9) o

2 El acompañamiento del Programa Binacional de Educación Migrante en Morelos permitió el acceso y la identificación de los niños con experiencia migratoria en Estados Unidos. Los nombres de las escuelas y de los niños entrevistados se mantendrá en reserva para asegurar la confidencialidad de sus identidades. 
el tercer $(6)^{4}$ año. Con excepción de un niño nacido en México (en Guerrero), los demás alumnos entrevistados nacieron en Estados Unidos. Tanto en las entrevistas como en el análisis de la Encuesta Intercensal, los niños con trayectoria migratoria de retorno aparecen poco, es decir, es una trayectoria migratoria difícil de encontrar. Incluso a nivel nacional, solo el $5.8 \%$ de los menores con experiencia migratoria son retornados en 2015. En Cuernavaca, en cierta forma, se reproduce lo observado a nivel nacional. Sin embargo, el panorama a nivel del estado de Morelos es distinto; ahí el porcentaje de población retornada asciende a $45.2 \%$, la proporción de inmigrantes con cinco años o más en México es de $45 \%$ y solo $9.8 \%$ son menores inmigrantes recientes, es decir, nacidos en Estados Unidos con cinco años o menos viviendo en México. Este es un resultado interesante de analizar en trabajos futuros ya que deja ver un patrón de migración diferenciado en Morelos con respecto al promedio nacional e incluso a la capital del estado.

De esta manera, entre los entrevistados predominan los niños nacidos en Estados Unidos, ya sean migrantes recientes o de larga data, lo que da como resultado que tengan distintas trayectorias migratorias, definidas por el tiempo de residencia en México. Antes de ir a fondo en lo referente a entrelazar la trayectoria migratoria con la trayectoria escolar conviene describir algunas características de los niños entrevistados.

Del total de entrevistados, 25 son nacidos en Estados Unidos y uno en México, en Guerrero para ser preciso. Son 11 estados de la Unión Americana los que están representados; destacan Illinois y California, de estos dos estados proviene la mitad de los entrevistados. La otra mitad proviene de estados como Texas, Arizona, Carolina del Norte, Michigan, Missouri e Indiana, entre otros. Sin duda esto es reflejo de la diversidad de destinos que los migrantes han elegido para establecerse en Estados Unidos, cuestión que ha sido ampliamente documentada (López, 2015). ${ }^{5}$ La movilidad internacional de estos niños está ligada a la movilidad internacional de sus padres, por ello los diversos orígenes son el reflejo de la movilidad que ellos llevaron a cabo en un inicio. En cuanto a los lugares de residencia al momento de la entrevista, con excepción de dos niños, ${ }^{6}$ todos vivían en Cuernavaca.

Ahora bien, el análisis de las entrevistas se hace a través de una herramienta como la trayectoria. Esta "permite realizar un recorte analítico de la biografía, ordenar, sistematizar e interpretar la experiencia migratoria en un intervalo de

4 Se llevaron a cabo un total de 30 entrevistas, aunque cuatro de ellas fueron incompletas.

5 De los 11.5 millones de personas que viven en Estados Unidos y nacieron en México, $51.1 \%$ se ubica en la región Oeste, 33.8 \% en la región Sur, 11 \% en la región del Medio Oeste y 4.1 \% en la región Noreste.

6 Uno vivía en el municipio de Temixco y otro en el de Emiliano Zapata, ambos colindantes con el de Cuernavaca. 
tiempo, condensando las imbricaciones entre las condiciones históricas de un sujeto migrante y la experiencia migratoria de la persona" (Rivera, 2012, p. 455). Reconstruir la trayectoria migratoria implicó preguntar sobre el lugar de nacimiento, el lugar de residencia actual y los movimientos previos (en Estados Unidos y México) a su última llegada a México. En esta forma se delinea el número total de movimientos migratorios (internos e internacionales) que han realizado los menores. Aunado a ello, se preguntó acerca del inicio de la vida escolar para aproximarse a la trayectoria educativa; en qué país se inició, México o Estados Unidos; además de indagar sobre si han repetido año y otros detalles más cercanos a su proceso de integración escolar tales como su sentir al llegar a la escuela en México, las burlas, el apoyo en tareas y un punto central, el idioma. De esta manera, la trayectoria "puede contribuir a entender la dinámica de múltiples movilidades insertas en los procesos migratorios contemporáneos" (Rivera, 2012, p. 460). Así, desde la postura cualitativa asumida en esta investigación se busca "explicar la interconexión de los eventos y los hechos ocurridos en un intervalo, comprender el significado que el individuo otorga a los eventos o episodios biográficos, relatados en una entrevista" (Rivera, 2012, p. 462). El análisis de la trayectoria migratoria y educativa arroja lo que se analiza en las subsecciones siguientes.

\section{Las trayectorias migratorias y educativas en Cuernavaca}

Salvo Daniel, que nació en Guerrero, México, y se fue a los seis años a San Diego, California, junto con sus padres, todos los demás entrevistados tuvieron como punto de partida Estados Unidos. La edad de la última llegada a México varía entre los entrevistados, desde Yumairi que llegó a los tres meses hasta Nacho que arribó a Cuernavaca a los 10 años. Sin duda esto hace que estemos ante un rango amplio de edad a la última llegada y, por consiguiente, que los años que llevan viviendo en México vayan desde uno hasta casi 14 años. Este amplio rango en el tiempo que llevan viviendo en México implica que, de manera similar a lo que se hizo en el análisis de la base de datos de la Encuesta Intercensal, se haga una agrupación de los menores entrevistados de acuerdo con la edad de llegada a México (o bien los años que llevan viviendo en el país). La importancia de realizar esta tipología se relaciona con la propuesta de Rumbaut (2004) analizada en el apartado anterior, esto es, la edad de llegada al nuevo destino importa y tiene efectos en el proceso de integración. De esta manera, se identifica a seis menores entrevistados que tienen entre dos y seis años viviendo en México, es decir, son de entre todos los entrevistados los que menos tiempo llevan viviendo en el país. Cinco más tienen entre siete y nueve años viviendo en México y los 15 restantes tienen entre 10 y 14 años en el país. En esta forma, se identifican tres tiempos de llegada a México: uno reciente, uno de larga data y otro 
intermedio (esquema 3). Esta tipología permite perfilar en dónde fue el inicio de la trayectoria escolar - por supuesto se preguntó directamente acerca de ello- dado que son menores de entre 12 y 15 años y más de la mitad han vivido en México 10 años o más, es decir, iniciaron la escuela en México. A partir de esta propuesta de tipología de acuerdo con el tiempo de llegada se presentan tres casos, uno representativo de cada trayectoria migratoria, esto es, un niño de llegada reciente, uno de llegada intermedia y otro con largo tiempo de llegada. Esto ayudará a mostrar las diferencias en el proceso de integración escolar que se está analizando.

En cuanto a la trayectoria escolar, estrechamente relacionada con la trayectoria migratoria, no se observan diferencias en cuanto al tiempo de llegada; por lo tanto, no se lleva a cabo tipología alguna debido a que son trayectorias educativas que no se han visto interrumpidas, independientemente del tiempo de llegada a México. Por último, respecto a los elementos que ayudan a entender el proceso de integración escolar de los menores, el idioma juega un papel central en este proceso. En los tres casos que a continuación se presentan se analiza la trayectoria migratoria, la escolar y el proceso de integración.

ESQUEMA 3. Definición de las trayectorias migratorias de acuerdo con el tiempo que llevan viviendo en México

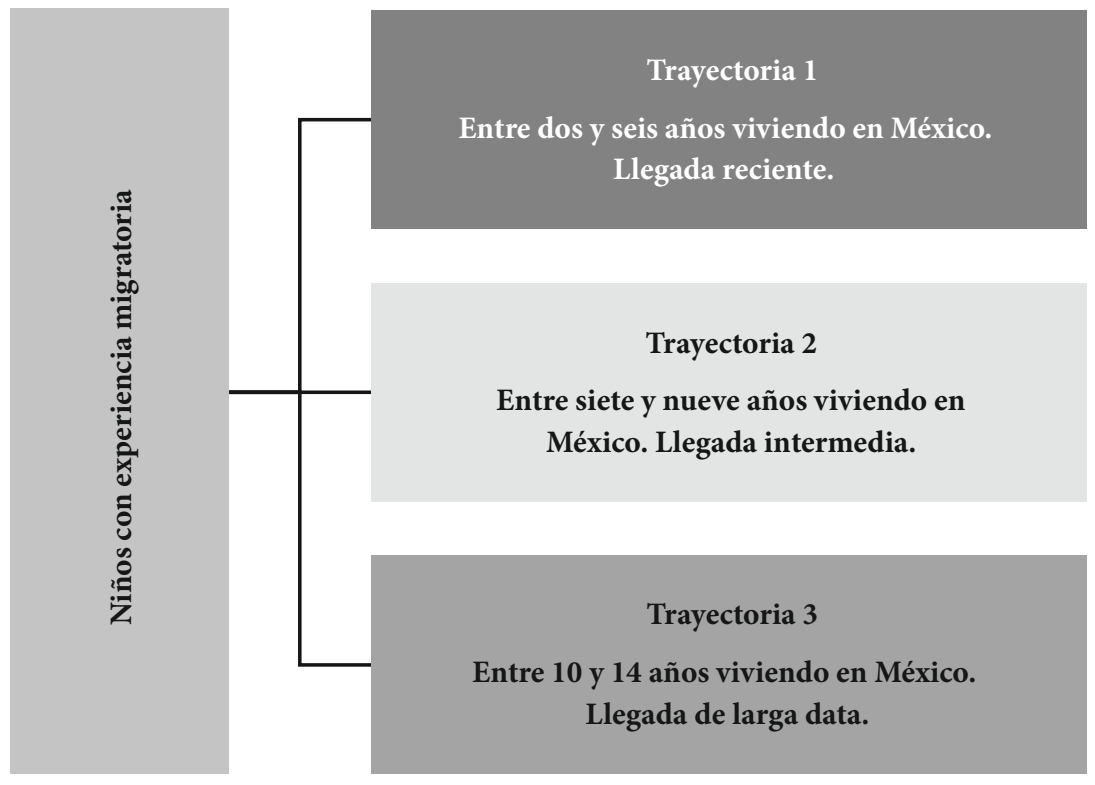

Fuente: Elaboración propia. 


\section{Nacho. Llegada reciente}

Nacho nació en San Diego, California, al momento de la entrevista tenía 12 años y estudiaba el primero de secundaria en una escuela del norte de la capital del estado de Morelos. Llegó a Cuernavaca a los 10 años, es decir, tenía aproximadamente dos años viviendo en México, por lo que la experiencia de su proceso de integración escolar es reciente. Sin embargo, su última llegada a México no es su único movimiento internacional. En la reconstrucción de su trayectoria migratoria se detecta que él había estado en México cuando tenía dos años de edad, aunque volvió a Estados Unidos y vivió allá alrededor de nueve años. De esta manera, a su corta edad, Nacho ha realizado tres movimientos migratorios con la intención de vivir entre México y Estados Unidos. Podemos decir que él tiene una llegada reciente a México y, además, la suya se puede caracterizar como una trayectoria migratoria de alta movilidad. $^{7}$

Nacho no vio afectado el inicio de su trayectoria escolar, que empezó en Estados Unidos, debido a que su primer movimiento migratorio entre ambos países lo hizo a los dos años. Cursó hasta el cuarto año de elementary school en Estados Unidos, por lo tanto estuvo expuesto a ese sistema educativo al menos cuatro años - sin contar el pre kínder y el kínder-. Inició el quinto año de primaria en Cuernavaca, su trayectoria escolar no registra repetición de curso y lleva dos años estudiando en la ciudad: el quinto y sexto año de primaria y parte de primero de secundaria. Aun cuando la última migración no afectó su trayectoria escolar, Nacho menciona que sintió difícil su llegada a la escuela en Cuernavaca (parte de su proceso de integración escolar) principalmente porque al momento de su arribo hablaba inglés, lo que le hizo difícil su integración. Este obstáculo propició que, con el apoyo familiar, tomara clases particulares de español durante dos meses. Nacho dijo que cuenta con doble nacionalidad y entre sus expectativas futuras está regresar a Estados Unidos a estudiar y trabajar, esta expectativa está influenciada por el hecho que tiene un hermano mayor que estaba próximo a irse a Estados Unidos.

\section{Edy. Llegada intermedia}

Edy nació en Santa Ana, California. Tenía 13 años al momento de la entrevista y cursaba el segundo año de secundaria; su lugar de residencia era Cuernavaca,

La trayectoria de alta movilidad se define así por el número de movimientos migratorios que los menores han realizado a lo largo de su vida. En este caso es alta cuando se tienen tres o más movimientos migratorios nacionales e internacionales. Una trayectoria de baja movilidad estaría definida en función de un solo movimiento migratorio internacional. 
Morelos. Edy llegó a esa ciudad cuando tenía seis años, por lo que llevaba siete viviendo ahí. La última llegada a México de Edy no es su único movimiento migratorio entre México y Estados Unidos. Él regresó a los nueve meses a México, pero solo estuvo alrededor de dos meses y volvió a Estados Unidos. No regresó a México sino hasta los seis años. En Estados Unidos vivía con su madre y su padre, en México solo vive con su madre. Al igual que Nacho, Edy ha realizado tres movimientos migratorios, por lo que su trayectoria se considera de alta movilidad.

La trayectoria escolar de Edy no ha sido afectada por los movimientos migratorios, es decir, no ha experimentado repetición de años. Esto se explica porque cuando realizó el primer viaje no estaba en edad escolar, por lo tanto solo uno de los viajes - el último- coincide con que él estuviera en edad escolar; sin embargo, tampoco lo afectó porque coincidió con su transición entre el preescolar y la primaria. Inclusive, al indagar en su trayectoria escolar, resulta que él inició el preschool en Estados Unidos y le faltó poco para culminarlo; fue entonces cuando regresó a Cuernavaca a terminar el kínder, e inició la primaria en esa ciudad. Edy menciona que su llegada a la escuela en Cuernavaca fue fácil; el hecho de que hablaba español ayudó en su proceso de integración, y el tiempo que estuvo expuesto al sistema escolar estadounidense fue relativamente pequeño. También menciona que el inglés se le olvidó, lo cual se explica por el tiempo que lleva viviendo en Cuernavaca —ocho años-. Edy dice tener la doble nacionalidad y entre sus expectativas está volver a Estados Unidos con la intención de estudiar.

\section{Mariana. Llegada de larga data}

Mariana es el caso elegido para ejemplificar una trayectoria migratoria de larga data. Ella nació en Salinas, California. Al momento de la entrevista cursaba el segundo de secundaria y tenía 14 años. Llegó a Cuernavaca cuando tenía nueve meses y desde entonces no ha vuelto a vivir en Estados Unidos; sin embargo, en su trayectoria migratoria se detecta un movimiento migratorio interno, hacia Tijuana; no recuerda la edad que tenía cuando vivió en aquella ciudad fronteriza. Ella ha realizado tres movimientos migratorios, uno internacional y dos nacionales, por lo que su trayectoria puede ser catalogada como de alta movilidad. Aun cuando ha tenido alta movilidad su trayectoria escolar no ha sido afectada por estos movimientos; la explicación a esto la encontramos en la edad muy temprana en la que se llevaron a cabo dichos movimientos.

La trayectoria escolar de Mariana se inició en México, por lo que lleva ya ocho años estudiando en Cuernavaca; ella no registra repetición de años. Mariana dice que no habla inglés, pero lo entiende; dice no tener doble nacionalidad y, aun cuando no tuvo experiencia escolar en Estado Unidos, sabe que en las escuelas estadouni- 
denses hay casilleros, gimnasio y bebederos. Tiene la expectativa de volver a Estados Unidos con la intención de terminar sus estudios (high school) y conocer aquel país. Hay que recordar que ella llegó a México cuando tenía nueve meses.

El hecho de mostrar aquí tres casos con tres distintas trayectorias migratorias tiene el objetivo de presentar las diferencias y similitudes que enfrentan los menores con experiencia migratoria en su proceso de integración en las escuelas mexicanas, específicamente en escuelas de Cuernavaca. En términos generales, considerando todas las entrevistas y no solamente los tres casos presentados, quienes estuvieron más expuestos al sistema escolar en Estados Unidos enfrentaron dificultades al incorporarse en las escuelas de Cuernavaca. De esta manera, podemos sugerir que sí hay diferencia en el proceso de integración escolar en función de la trayectoria migratoria de los menores, quienes tienen una llegada reciente enfrentan más obstáculos que quienes tienen una trayectoria migratoria intermedia o de larga data. El principal obstáculo para los menores de arribo reciente se relaciona con el idioma. Ejemplo de ello es lo que mencionan otros menores en las entrevistas, "mezclaba inglés con español” (Ximena, secundaria 22), "me sentí rara, no sabía nada, no entendía a los maestros, me costaba trabajo traducir las palabras" (Isamar, secundaria 44), "hablaba más inglés (que español)" (Odalys, secundaria 9).

Por otro lado, independientemente del tiempo que llevan viviendo en Cuernavaca, ninguno de los entrevistados ha repetido años. En esto no hay diferencias entre trayectorias migratorias. De hecho se esperaba que entre quienes llevaron a cabo varios movimientos migratorios la discontinuidad escolar sería un elemento que considerar porque la migración rompe la cotidianidad y, por ende, con ciertas continuidades, como lo puede ser la vida escolar; sin embargo, la migración no ha tenido tal efecto en la trayectoria escolar. Esto podría deberse a que los movimientos migratorios fueron a una edad temprana (trayectoria reciente e intermedia), cuando incluso aún no se iniciaba la vida escolar (trayectoria de larga data).

Un punto que no se debe dejar de lado es el relacionado con la expectativa de ellos de volver a Estados Unidos; solo uno de los menores nació en Guerrero (Daniel, secundaria 44), todos los demás nacieron en aquel país. Únicamente cinco de ellos declararon que no volverían a Estado Unidos, el resto dijo que sí lo haría. Las razones que expusieron se pueden agrupar en dos: 1) ir a estudiar (o terminar sus estudios), y 2) reunirse con sus familiares que aún están en Estados Unidos.

Este panorama es el que se observó en cuatro escuelas secundarias de Cuernavaca, Morelos. Pasemos ahora al último apartado. 


\section{Reflexiones finales}

En este artículo se propuso como objetivo analizar el proceso de integración escolar de niños con experiencia migratoria en Estados Unidos que en 2016 residían en Cuernavaca, Morelos. El análisis se hizo mediante una serie de entrevistas; a partir de las respuestas se analizó la trayectoria migratoria y la trayectoria educativa, como ejes principales que permitieron entender el proceso de integración escolar de los menores.

Los resultados más notables que se identifican en las cuatro secundarias de Cuernavaca son los siguientes. El primero de ellos es que las trayectorias migratorias no son homogéneas y predominan aquellas que son de larga data; es decir, si bien los menores con experiencia migratoria nacieron en Estados Unidos, hicieron la migración a muy temprana edad. De acuerdo con la propuesta de Rumbaut (2004) ellos serían catalogados como generación 1.75 por su gran parecido a la segunda generación de inmigrantes, en la presente investigación ellos serían más parecidos a los menores que viven en Cuernavaca y no experimentaron movimiento migratorio alguno. Una de las principales características de los menores con trayectoria migratoria de larga data es que iniciaron su vida escolar en México, específicamente en Cuernavaca; por lo tanto, los posibles efectos negativos de experimentar la migración no son claros, al menos no en la repetición de años ni en el proceso de integración escolar, en el cual no hubo dificultades, al menos no asociadas a la migración.

Las trayectorias migratorias recientes e intermedias comparten ciertas características; una de ellas es que los menores iniciaron su vida escolar en Estados Unidos, en espacios como el kínder, el preschool o el elementary school. Aun cuando su llegada a Cuernavaca fue luego de que iniciaron la vida escolar en Estados Unidos, ninguno de estos menores repitió años. Lo que sí se hizo evidente en sus respuestas es la dificultad que experimentaron en su ingreso a la escuela principalmente por el idioma, ellos hablaban inglés y las clases eran en español. En términos teóricos, guiándonos por la propuesta de Rumbaut (2004), estos menores con experiencia migratoria serían de la generación 1.5, es decir, menos parecidos a aquellos menores que no migraron.

Distinguir las trayectorias migratorias a partir del tiempo de estancia en México permitió evaluar los posibles efectos negativos de la migración en el proceso de integración escolar y con ello demostrar la estrecha relación entre la edad de llegada y la integración escolar, en este caso, en un contexto de migración de retorno; en esta forma, mientras más temprano se migré, menos dificultades se enfrentan en el proceso de integración escolar. Para las autoridades escolares este resultado es fundamental, sobre todo en los municipios, localidades y escuelas con importante recepción de menores con experiencia migratoria. Los hallazgos presentados 
complementan la información recabada por las autoridades escolares por medio del formato $911,{ }^{8}$ en el que se registran, entre otros datos, el lugar de nacimiento y el de procedencia (en caso de venir de una escuela de otro país) de todos los estudiantes. Es decir, se cuenta con información general complementada con el análisis puntual de las trayectorias de los menores con experiencia migratoria para detectar y, en su caso, implementar medidas que faciliten el proceso de integración académica y cultural de los menores.

Los resultados indican que la migración por sí misma no afecta la trayectoria escolar de los menores entrevistados, al menos esto no se refleja en la repetición de años. Si bien este es un elemento positivo, también es un hecho que existen necesidades específicas por atender entre esta población. Precisamente, esta investigación ayuda a diferenciar estas necesidades de apoyo, es decir, que no todos los menores entrevistados requieren superar los mismos obstáculos. Por ejemplo, entre aquellos menores que llevan más tiempo viviendo y asistiendo a las escuelas en Cuernavaca el proceso de integración escolar no parece ser una barrera, dado que la mayoría de ellos iniciaron su vida escolar en la ciudad, por lo tanto no transitaron por el proceso de llegar de una escuela en Estados Unidos a una nueva en México. Sin embargo, un número importante de estos menores no tienen doble nacionalidad, es decir, solo cuentan con papeles que demuestran que han nacido en Estados Unidos y no en México. Este, sin duda, es un punto que deben considerar las autoridades educativas porque la falta de identidad legal en México podría convertirse en una barrera que les impida continuar sus estudios; en específico, en el sistema educación media superior se solicita como requisito contar con la Clave Única de Registro de Población (CURP), para lo cual es necesario contar con la doble nacionalidad o tener el documento migratorio expedido por el Instituto Nacional de Migración que avale su estatus migratorio en México. Un segundo ejemplo lo podemos ubicar entre aquellos que tienen menos tiempo residiendo en Cuernavaca. La mayoría de ellos cuenta con doble nacionalidad, por lo que esto no será un obstáculo que interfiera en su futura trayectoria escolar. Sin embargo, entre estos menores el proceso de integración escolar sí es un elemento que se debe considerar, en especial porque no hay un apoyo puntual de las escuelas en cuanto a solventar la barrera del idioma. Ellos llegan hablando inglés y las clases son en español.

Por último, las expectativas futuras de los menores con experiencia migratoria implican un reto presente para las escuelas en ambos países, México y Estados Unidos. Este reto tiene que ver con el idioma. Gran parte de los entrevistados mencionaron que desearían volver a Estados Unidos - cierto que es una expectati- 
va, y también es cierto que son nacionales de aquel país - a continuar sus estudios y a reunirse con sus familiares que aún viven allá. Quienes llevan más tiempo en México ya no hablan inglés, según lo declararon ellos mismos. Entre quienes llevan menos tiempo es variado; unos sí lo hablan, otros no; sin embargo, al no estar expuestos al idioma inglés este se podría ir perdiendo con el paso del tiempo, justo como pasó con aquellos que llevan más tiempo en México. En materia de política educativa, se identifican al menos dos esfuerzos por combatir el rezago en inglés, uno a nivel estatal y otro a nivel federal. El primero es un proyecto del Sistema Educativo Estatal de Baja California (2016) en el cual "se impulsa el uso del idioma inglés con enfoque conversacional en los plateles educativos de todos los niveles..." con el objetivo de buscar que tenga un uso cotidiano dentro y fuera del aula. A nivel federal, el Nuevo Modelo Educativo contempla la Estrategia Nacional de Inglés, la cual tiene por objetivos la formación de maestros para que tengan un adecuado manejo de este idioma y el inglés para todos a través de la modificación y adaptación de los programas de estudio de acuerdo con las necesidades de los alumnos (Secretaría de Educación Pública, 2017). Si bien estos programas son esfuerzos por subsanar un rezago en materia de idioma inglés, se necesita tiempo para que los resultados se observen, en el entendido de que la aplicación de este tipo de medidas contempla el aprendizaje de los alumnos y el aprendizaje y certificación de otra lengua por parte de los profesores. Por esta razón, si los niños no alcanzan a ser beneficiarios de este tipo de programas y ya no hablan inglés, cuando decidan regresar a su país de nacimiento a continuar sus estudios ¿en qué condiciones lo harán? ¿Qué se hace en las escuelas en México y Estados Unidos? ¿La paradoja está ahí, estadounidenses sin oportunidades? Estas preguntas estarán presentes en un futuro cercano.

\section{Referencias}

Aguilar, R. (2014). "Nos regresamos pa'tras"”. Diferencias en el desempeño escolar de niños y jóvenes en un contexto de migración de retorno". Tesis de doctorado en Estudios de Población, Centro de Estudios Demográficos, Ambientales y Urbanos: El Colegio de México. Ciudad de México.

Jacobo, M. (2017). De regreso a "casa" y sin apostilla: estudiantes Méxicoamericanos en México. Sinéctica, 48, 118.

Giorguli, S. y Gutiérrez, E. (2011). Niños y jóvenes en el contexto de la migración internacional entre México y Estados Unidos. Coyuntura Demográfica, 1, 21-25.

Instituto Nacional para la Evaluación de la Educación (INEE) (2016). Panorama educativo de México 2015. Indicadores del Sistema Educativo Nacional. Educación básica y media superior. México: INEE. 
Instituto Nacional de Estadística y Geografía (INEGI) (2015). Encuesta Intercensal 2015. Aguascalientes: INEGI.

López, G. (2015). Hispanics of Mexican origin in the United States, 2013. Washington: Pew Research Center.

López Castro, G. (2007). Niños, socialización y migración a Estados Unidos. En Ariza, M. \& Portes, A. (coords.), El país transnacional: migración mexicana y cambio social a través de la frontera (pp. 545-570). México: Instituto de Investigaciones Sociales-Universidad Nacional Autónoma de México (UNAM).

López Castro, G. (1999). La educación en la experiencia migratoria de los niños migrantes. En Mummert, G. (ed.), Fronteras fragmentadas (pp. 359-374). México: El Colegio de Michoacán, Centro de Investigación y Desarrollo del estado de Michoacán.

Oropesa, R. S. \& Landale, N. S. (1997). In search of the new second generation: alternative strategies for identifying second generation children and understanding their acquisition of English. Sociological Perspectives, 40(3), 427- 455.

Passel, J. \& Cohn, D. (2017). As Mexican share declined: U.S. unauthorized immigrant population fell in 2015 below recession level. Washington: Pew Research Center.

Portes, A. \& Zhou, M. (1993). The new second generation: segmented assimilation and its variants. Annals of the American Academy of Political and Social Science, 530(1), 74-96.

Rivera, L. (2012). Las trayectorias en los estudios de migración: una herramienta para el análisis longitudinal cualitativo. En Ariza, M. \& y Velasco, L. (coords.), Métodos cualitativos y su aplicación empírica: por los caminos de la investigación sobre migración internacional. México: Instituto de Investigaciones SocialesUNAM, El Colegio de la Frontera Norte.

Rumbaut, R. (2004). Ages, life stages, and generational cohorts: decomposing the immigrant first and second generations in the United States. International Migration Review, 38(3), 1160-1205.

Sánchez García, J. \& Zúñiga, V. (2010). Trayectorias de los alumnos transnacionales en México. Propuesta intercultural de atención educativa. Trayectorias, 12(30), 5-23.

Secretaría de Educación Pública (2017). Página oficial, http:// docs.google.com/ gview?url=https://www.gob.mx/cms/uploads/attachment/file/240633/1._Resumen_Ejecutivo_6_.pdf (consultada el 28 de diciembre de 2017).

Sistema Educativo Estatal de Baja California (2016). Página oficial, http://www. .educacionbc.edu.mx/eventos/2016/EncuentroIngles/Encuentro\%20Academic o\%20De\%20Ingles\%20Conversacional.pdf (consultada el 28 de diciembre de 2017). 
Vargas, E. \& Camacho, E. (2015). ¿Cambiarse de escuela? Inasistencia y rezago escolar de los niños de migración reciente de Estados Unidos a México. Norteamérica, 2, 157-186.

Zúñiga, V. \& Hamann, E. (2008). Escuelas nacionales, alumnos transnacionales: la migración México/Estados Unidos como fenómeno escolar. Estudios Sociológicos, XXVI(76), 65-85. 